\title{
BMJ Open Recruitment strategies in randomised controlled trials of men aged 50 years and older: a systematic review
}

\author{
Karen Bracken, ${ }^{\oplus 1}$ Lisa Askie, ${ }^{1}$ Anthony C Keech, ${ }^{1}$ Wendy Hague, ${ }^{1}$ Gary Wittert ${ }^{2}$
}

To cite: Bracken K, Askie L, Keech AC, et al. Recruitment strategies in randomised controlled trials of men aged 50 years and older: a systematic review. BMJ Open 2019:9:e025580. doi:10.1136/ bmjopen-2018-025580

- Prepublication history and additional material for this paper are available online. To view please visit the journal (http:// dx.doi.org/10.1136/bmjopen2018-025580).

Received 23 July 2018 Revised 17 December 2018 Accepted 13 February 2019

Q Check for updates

(c) Author(s) (or their employer(s)) 2019. Re-use permitted under CC BY-NC. No commercial re-use. See rights and permissions. Published by BMJ.

${ }^{1}$ NHMRC Clinical Trials Centre, Camperdown, New South Wales, Australia

${ }^{2}$ Department of Medicine, University of Adelaide, Adelaide, South Australia, Australia

Correspondence to

Karen Bracken;

karen.bracken@ctc.usyd.edu.au

\section{ABSTRACT}

Objectives To identify and review evaluations of strategies to recruit men aged 50 years and over to randomised controlled trials (RCTs).

Design Systematic review and narrative synthesis. Data sources MEDLINE, EMBASE, CINAHL and ORRCA databases were searched to 1 December 2017. Eligibility criteria Studies using quantitative methods to evaluate recruitment strategies to RCTs of men aged 50 years and older.

Data extraction and synthesis A single reviewer extracted data (for each strategy, number of participants approached, screened and randomised, and cost). Study quality was assessed using National Heart, Lung and Blood Institute Quality Assessment Tools and considered study design, description of interventions, description and measurement of outcomes, completeness of outcome reporting, performance of statistical testing and consideration of confounders. Recruitment strategies were categorised by the recruitment stage they addressed. Results Sixteen studies ( $\mathrm{n}>14000)$ were included: one good quality, ten fair quality and five poor quality. Studies evaluated strategies to identify prospective participants, and to improve the processes for assessing participant eligibility, providing participant information and seeking consent. In good and fair quality studies, the most effective strategies for identifying participants were referral from an affiliated health service provider (two studies), mass mailing (five studies) and media coverage (two studies). Community outreach activities such as displaying posters and attending local community events were not effective (two studies). Trial-specific training of site recruitment staff, developed using qualitative analysis of recruitment visits (two studies), and provision of study information to prospective participants at a multidisciplinary, group information session (one study) both improved recruitment. Conclusion Improved engagement of men aged 50 years and older in RCTs is needed. A gender-sensitised approach to RCT recruitment may help to address this need. We have identified several promising recruitment strategies that merit further evaluation.

PROSPERO registration number CRD42017060301.

\section{INTRODUCTION}

Randomised controlled trials (RCTs) are the accepted gold standard in health intervention research. Recruitment to RCTs can be challenging, and around $50 \%$ of RCTs fail
Strengths and limitations of this study

This review incorporated systematic database search strategies and quality assessment tools to identify and appraise eligible studies.

- The categorisation of included studies according to the stage of the recruitment pathway they addressed is a practical approach designed to aid interpretation of the review results by trial managers.

- Many of the included studies were at risk of significant or some bias, limiting the reliability of the results presented in these papers.

- Few studies reported the cost of recruitment strategies.

to achieve their recruitment targets. ${ }^{1-3}$ The potential consequences of failed RCT recruitment are considerable and include wasted research resources, delays in the release of RCT results and increased likelihood of type 2 error. RCTs expose trial participants to potential risk and inconvenience, and trials that fail to recruit fully may waste the goodwill and commitment of the participants that they do recruit. Clinical trial unit directors have identified the evaluation of strategies to boost recruitment as the highest priority in trial methodology research. ${ }^{4}$

Despite the importance of successful recruitment to the overall success of trials and the calls for research in this area, the published evidence on how best to conduct RCT recruitment is limited. ${ }^{5}$ Several large systematic reviews have found surprisingly few randomised evaluations of recruitment strategies with many randomised recruitment studies being underpowered, low quality or set within hypothetical rather than real-world RCTs. ${ }^{6-8}$ Other recent recruitment-focused systematic reviews have concentrated on specific demographic groups or disease areas. ${ }^{9-14}$ This approach recognises the diversity of trial populations, interventions and designs to build a greater understanding of how recruitment strategies may influence specific participant groups. ${ }^{15}$ 
It is well-established that the differences in disease incidence and health outcomes observed in men and women are determined not only by biological sex differences but also by socially constructed gender roles and norms. There is an increasing focus on gender-sensitived health service delivery to address health inequities for both men and women. ${ }^{16}{ }^{17}$ Women have been historically under-represented in clinical trials, and so gendered approaches to trial recruitment have often focused on the recruitment of women. ${ }^{1819}$ However, research is also needed to better engage men in clinical trials. Men have a lower life expectancy than women, and men, especially those aged over 50 years, bear a greater disease burden. ${ }^{20}{ }^{21}$ In the past, men have been characterised as disengaged with healthcare services but it is now recognised that men will engage willingly and effectively with healthcare that recognises, and is tailored to, men's preferences. ${ }^{22}{ }^{23}$ An exploration of gender-sensitised strategies to recruit men to RCTs may, therefore, be worthwhile, ${ }^{24}$ particularly since men may be under-represented in RCTs of disease prevention ${ }^{25}$ and health promotion. ${ }^{2627}$

Evaluations of online and social media recruitment strategies are becoming more common with promising results reported in the recruitment of adolescents and young people ${ }^{1028}$ and women. ${ }^{2930}$ Facebook and other types of online promotion may achieve a broader reach and be more cost-effective than traditional recruitment methods such as newspaper advertising, media coverage and posters. ${ }^{30}{ }^{31}$ However, a recent systematic review of recruitment using Facebook found little evidence of its effectiveness in recruiting participants aged over 35 years. ${ }^{28}$ It is therefore unclear whether online and social media strategies are effective in recruiting men aged over 50 to RCTs.

This review aims to identify and review evaluations of strategies to recruit men aged over 50 years to RCTs in order to guide recruitment planning for future men's health RCTs.

\section{METHODS}

\section{Eligibility criteria}

Studies met our inclusion criteria if they evaluated a strategy or strategies intended to improve the recruitment of men aged 50 years or older to an RCT. Studies must have reported at least one of the defined, quantitative, recruitment outcome measures. Studies were eligible irrespective of whether they recruited patients or healthy volunteers.

An initial scoping of the literature revealed that recruitment studies set within RCTs of both men and women often failed to provide adequate detail to determine the effectiveness of recruitment strategies on male participants alone. Therefore, to assess the impact of recruitment strategies on men, studies were only eligible for inclusion if set within an RCT recruiting men only.

The review included RCTs recruiting participants aged 50 years and older. Where the age range was not specified, studies were included where the mean/median age was 60 years or older, or where the disease of interest was prevalent in older men (eg, prostate cancer).

Included studies needed to evaluate a specific recruitment strategy or strategies; papers describing barriers and facilitators to recruitment or discussing informed consent but not presenting a specific strategy or approach to recruitment were excluded. Similarly, papers providing a brief account of recruitment without describing or evaluating specific strategies or approaches were excluded.

The search strategy was restricted to papers published since 2000. Communication channels and data management practices are central to recruitment research. Both of these areas have been transformed in the past 18 years by the growth of internet access. Evaluations published before 2000 are therefore less likely to be relevant to current trial practices, particularly those reporting advertising and media-related strategies.

\section{Search strategy}

A search of four databases (Medline, Embase, CINAHL and ORRCA) was performed in July 2017 and updated in December 2017. Studies published in English from 2000 onwards were considered for inclusion. Individualised search strategies (available in online supplementary file 1) were developed for each database using a combination of keywords relating to recruitment, enrolment, men and RCTs. In addition, the reference lists of all included articles and other recruitment-related systematic reviews were searched by hand to identify other potentially relevant papers.

\section{Study selection and data extraction}

Citations and abstracts were exported to Endnote Version X8.2 and duplicates were removed. A $10 \%$ random sample of citations was selected for independent screening for eligibility by two reviewers ( $\mathrm{KB}$ and $\mathrm{GW}$ ), with disagreement resolved by discussion. The Kappa statistic for double-screened citations indicated substantial agreement (Kappa $=0.66)$ and the remaining $90 \%$ of articles were screened by $\mathrm{KB}$ alone.

Data from the included studies were extracted by KB using a prepiloted data extraction form. Studies were categorised according to disease area of the host RCT, type of host RCT (treatment, prevention or screening), number of participants in the recruitment study and recruitment study design. Where reported, the number of prospective participants who received the recruitment intervention and the number of those participants who went on to be screened and randomised to the host RCT were extracted. The costs incurred were also extracted.

\section{Categorisation of studies}

The Qualitative Research Integrated within Trials (QuinteT) group's Screened, Eligible, Approached, Randomised (SEAR) framework was developed to map each stage of the recruitment pathway. ${ }^{32}$ We adapted this framework to categorise the included studies according 


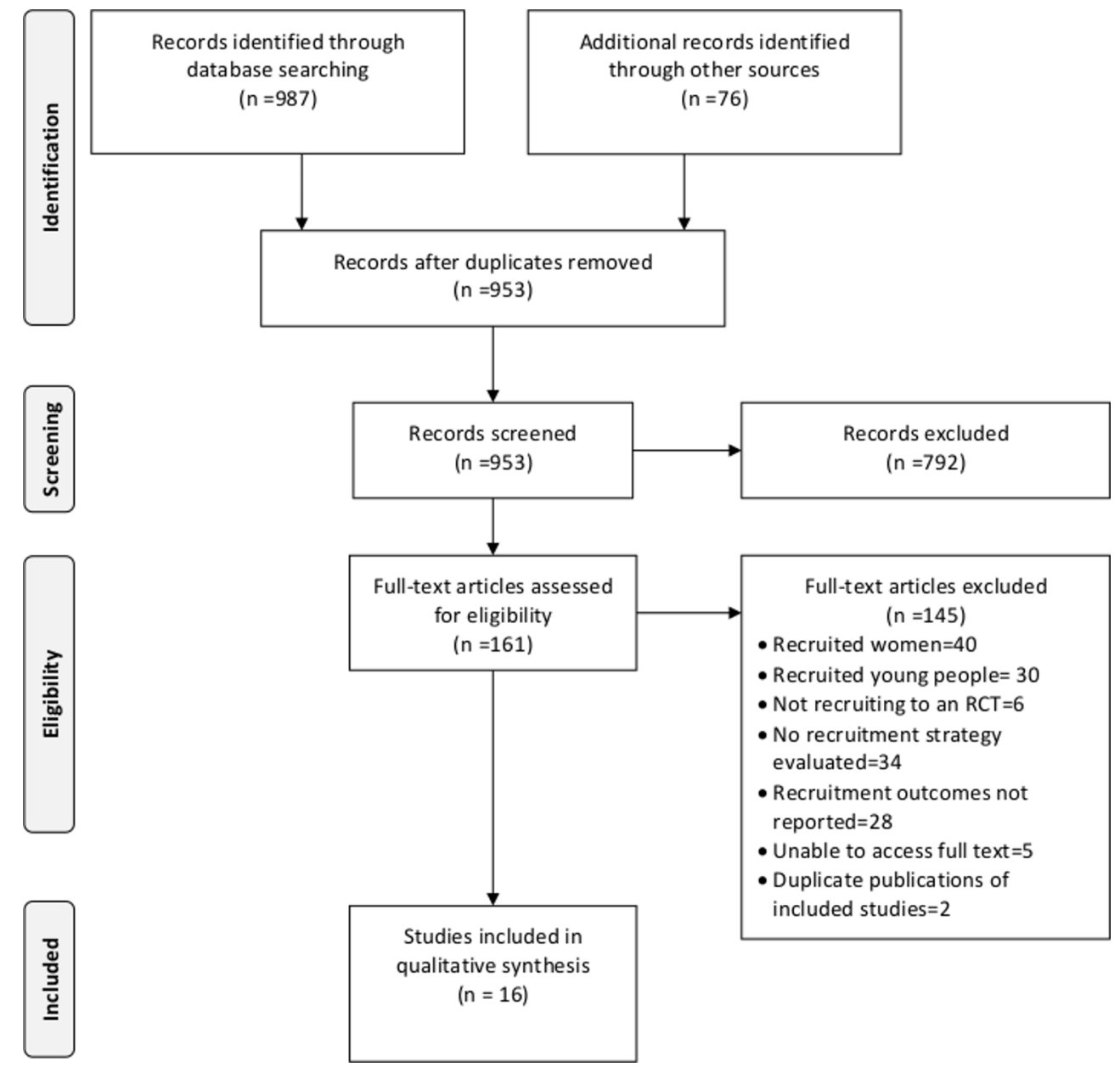

Figure 1 Search and screening results. RCT, randomised controlled trial.

to the stage or stages of the recruitment process they addressed: identification of participants ('Screened' in the SEAR framework), assessment of eligibility ('Eligible' in the SEAR framework) and patient information and consent ('Approached' in the SEAR framework).

\section{Outcome measures}

Our primary outcomes were: strategy uptake (defined as the percentage of people receiving the recruitment intervention who went on to be randomised to the host RCT), strategy contribution (defined as the percentage of all participants randomised to the host RCT who were randomised as a result of a particular strategy) and strategy cost (defined as direct or indirect cost per participant randomised).

\section{Assessment of study quality}

Six tools to assess study quality or risk of bias were identified from recent systematic reviews of recruitment strategies and were piloted for suitability and usability. After piloting, the National Heart, Lung and Blood Institute Quality Assessment Tools ${ }^{33}$ were selected as they addressed all included quantitative study designs, assessed key quality components and could be easily adapted for the assessment of non-clinical data. The tools listed criteria for judging study quality including study design, description of recruitment interventions, description and measurement of recruitment outcomes, completeness of outcome reporting, the performance of statistical testing and consideration of confounders. Based on these criteria, studies were subjectively judged by $\mathrm{KB}$, in consultation with $\mathrm{LA}$, as being of good (least risk of bias), fair (susceptible to bias) or poor (significant risk of bias) quality. Since this review addresses a methodological rather than a clinical question, the fair quality category was broadly defined to include studies that provided useful evaluation data even where some flaws were noted in the quality assessment. Quality assessments were performed with respect to the quantitative, recruitment-related outcomes of interest in this review only. The qualitative components of included mixed methods papers were not assessed as they were outside the scope of this review.

\section{Methods of analysis}

All studies, irrespective of quality, were included in the descriptive analysis in order to describe the full range of strategies evaluated and to assist with hypothesis generation for future research. Outcome measures were only analysed for studies of fair or good quality. Estimates from poor studies were excluded except where no estimates 
were available from studies of good or fair quality. In this case, the estimate is presented with a caveat that the study is of poor quality. We had planned to perform a meta-analysis if studies were sufficiently homogeneous in the target population and delivery of the intervention to do so.

The Preferred Reporting Items for Systematic Reviews and Meta-Analyses checklist was completed and can be found in online supplementary file 2 .

\section{Patient and public involvement}

Patients were not involved in the design or conduct of this systematic review. It is not possible to disseminate the results of this review to the participants of the included studies.

\section{RESULTS}

\section{Study selection}

Nine hundred and fifty-three unique papers were extracted. Of these, 16 recruitment studies were eligible for inclusion (figure 1). These 16 recruitment studies (listed in table 1) were conducted in the context of 12 RCTs since two RCTs hosted more than one recruitment study.

\section{Study characteristics}

The characteristics of included studies are described in table 2. As one might expect in trials recruiting exclusively older men, most selected studies reported recruitment to prostate cancer trials (11 studies plus one additional study in various cancers including prostate), with other studies reporting recruitment to trials in benign prostatic hyperplasia, low testosterone and suicide prevention. Three studies focused on the recruitment of men from minority ethnic groups. Recruitment studies ranged in size from 155 to 51085 screened participants and most commonly used a quantitative descriptive design.

\section{Quality assessment}

Most (10 of 16) studies were assessed as being of fair quality in relation to the recruitment outcomes of interest in this review. One study was evaluated as good, and five were evaluated as poor (tables 3-5). In general, all studies addressed a clear study question and enrolled a representative sample of participants. Recruitment outcomes were reliably measured and clearly reported, although few studies reported recruitment cost. However, the description and measurement of intervention delivery were often incomplete or missing. Some studies reported that interventions were delivered inconsistently across study sites, but this inconsistency was not accounted for in the reporting of outcomes. This limitation made comparisons within and between studies problematic. Possible confounding was also a common issue. Of the 16 recruitment studies, only three had a randomised design, and one additional, non-randomised study reported baseline demographic data by intervention group. In the remaining 12 studies, differences between the intervention groups in baseline characteristics could not be assessed. Therefore, differences in recruitment between groups may have been influenced by the characteristics of the individuals studied rather than the interventions evaluated. Furthermore, in some studies, several recruitment activities were implemented concurrently, but no study discussed the possible impact of this on the observed recruitment outcomes. Another common limitation was the lack of prospective study design. Three studies reported a prospective design; six reported a retrospective design and the remaining seven did not specify.

One study, which was otherwise of good quality, was assessed as fair due to inadequate sample size. ${ }^{34}$ Several studies incorporated both quantitative and qualitative designs, but were assessed for quality based on only quantitative analysis and outcomes. ${ }^{35-37}$

\section{Stages of recruitment and associated outcomes}

The included studies addressed three recruitment stages: (1) identification of prospective participants, (2) assessment of eligibility and (3) provision of participant information combined with seeking of consent. The strategies addressing each stage of recruitment are summarised below along with their reported recruitment outcomes. Outcomes are shown in table 6 (studies that reported strategy uptake), table 7 (studies that reported strategy contribution) and table 8 (studies that reported strategy cost).

\section{Identification of prospective participants}

Participant identification strategies were evaluated in nine studies. ${ }^{38-46}$ Excluding poor quality studies, all studies reported the contribution of participant identification strategies to enrolment (shown in table 7) while only four studies reported strategy uptake (shown in table 6) and two studies reported strategy cost (table 8). Within the participant identification category, we further grouped strategies as mass mailings, media coverage and advertising, health service referrals or community outreach activities. This categorisation was adapted from previous recruitment research. ${ }^{478}$ The data from table 7 have been summarised in table 9 to aid comparison between studies. The most frequently evaluated strategy were mass mailings and community outreach strategies (seven studies). Media strategies were evaluated in six studies and health service referrals in five studies.

\section{Mass mailing}

Recruitment by mass mailing involved sending study information and a letter of invitation to the members of one or more acquired mailing lists. Seven studies sent postal invitations ${ }^{38-424445}$ and one study also sent email invitations. ${ }^{45}$ Mailing lists were obtained from a variety of sources including the Department of Veterans Affairs database, Department of Motor Vehicles database, home owner database, participant lists from previous health research, 
Table 1 Key characteristics, summary of findings and quality assessment of included studies

\begin{tabular}{|c|c|c|c|c|c|c|c|c|}
\hline Author, year & Host RCT & $\begin{array}{l}\text { Host RCT } \\
\text { therapeutic area }\end{array}$ & $\begin{array}{l}\text { Recruitment } \\
\text { stage studied }\end{array}$ & $\begin{array}{l}\text { Recruitment } \\
\text { study design }\end{array}$ & $\begin{array}{l}\text { Screened/eligible } \\
\text { /randomised* (n) }\end{array}$ & Intervention(s) & $\begin{array}{l}\text { Summary of } \\
\text { findings }\end{array}$ & $\begin{array}{l}\text { Quality } \\
\text { assessment } †\end{array}$ \\
\hline Bhar et al, $2013^{38}$ & Not specified & $\begin{array}{l}\text { Suicide } \\
\text { prevention }\end{array}$ & $\begin{array}{l}\text { Identification of } \\
\text { participants }\end{array}$ & $\begin{array}{l}\text { Quantitative } \\
\text { descriptive }\end{array}$ & $233 / 48 / 33$ & $\begin{array}{l}\text { Various mass } \\
\text { mailing and } \\
\text { health service } \\
\text { referral strategies. }\end{array}$ & $\begin{array}{l}\text { Seeking referrals } \\
\text { from a co- } \\
\text { investigator's } \\
\text { clinic was the } \\
\text { most effective } \\
\text { strategy and } \\
\text { also had the } \\
\text { highest uptake } \\
\text { rate. Seeking } \\
\text { referrals from } \\
\text { non-collaborating } \\
\text { health services } \\
\text { and mass } \\
\text { mailings were } \\
\text { not effective } \\
\text { strategies. }\end{array}$ & Fair \\
\hline $\begin{array}{l}\text { Cauley et al, } \\
2015^{39}\end{array}$ & $\mathrm{~T}$ trials & $\begin{array}{l}\text { Low testosterone } \\
\text { treatment }\end{array}$ & $\begin{array}{l}\text { Identification of } \\
\text { participants }\end{array}$ & $\begin{array}{l}\text { Quantitative } \\
\text { descriptive }\end{array}$ & $51,085 / 931 / 790$ & $\begin{array}{l}\text { Various mass } \\
\text { mailing, media } \\
\text { and community } \\
\text { outreach } \\
\text { strategies. }\end{array}$ & $\begin{array}{l}\text { Mass mailing was } \\
\text { the most effective } \\
\text { recruitment } \\
\text { strategy and was } \\
\text { also the lowest } \\
\text { cost per man } \\
\text { screened. TV, } \\
\text { radio and print } \\
\text { advertisements, } \\
\text { clinicaltrials.gov } \\
\text { listing, posters } \\
\text { and flyers and } \\
\text { presentations at } \\
\text { events resulted } \\
\text { in very few men } \\
\text { being screened. }\end{array}$ & Poor \\
\hline $\begin{array}{l}\text { Chlebowski et al, } \\
2010^{40}\end{array}$ & SELECT & $\begin{array}{l}\text { Prostate cancer } \\
\text { prevention }\end{array}$ & $\begin{array}{l}\text { Identification of } \\
\text { participants }\end{array}$ & $\begin{array}{l}\text { Quantitative } \\
\text { descriptive }\end{array}$ & 4022/NR/634 & $\begin{array}{l}\text { Mailing to male } \\
\text { home owners } \\
\text { vs mailing } \\
\text { to previous } \\
\text { female research } \\
\text { participant } \\
\text { spouses. }\end{array}$ & $\begin{array}{l}\text { Mailing previous } \\
\text { female research } \\
\text { participants' } \\
\text { spouses resulted } \\
\text { in higher } \\
\text { recruitment } \\
\text { uptake than } \\
\text { mailing men and } \\
\text { was also more } \\
\text { cost-effective. } \\
\text { Mailing women } \\
\text { contributed fewer } \\
\text { participants than } \\
\text { mailing men due } \\
\text { to the relatively } \\
\text { small size of the } \\
\text { past research } \\
\text { participant } \\
\text { mailing list. }\end{array}$ & Fair \\
\hline $\begin{array}{l}\text { Cook et al, } \\
2010^{41}\end{array}$ & SELECT & $\begin{array}{l}\text { Prostate cancer } \\
\text { prevention }\end{array}$ & $\begin{array}{l}\text { Identification of } \\
\text { participants }\end{array}$ & $\begin{array}{l}\text { Non-randomised } \\
\text { controlled trial }\end{array}$ & NR/NR/8532 & $\begin{array}{l}\text { Various site- } \\
\text { directed } \\
\text { minority-targeted } \\
\text { recruitment } \\
\text { strategies funded } \\
\text { by minority } \\
\text { recruitment } \\
\text { enhancement } \\
\text { grants. }\end{array}$ & $\begin{array}{l}\text { Sites awarded } \\
\text { grants increased } \\
\text { recruitment } \\
\text { of African- } \\
\text { American men } \\
\text { significantly more } \\
\text { than matched } \\
\text { comparison } \\
\text { sites. Overall } \\
\text { recruitment was } \\
\text { also increased at } \\
\text { grant sites. }\end{array}$ & Poor \\
\hline $\begin{array}{l}\text { Heiney et al, } \\
2010^{42}\end{array}$ & EASE & $\begin{array}{l}\text { Prostate cancer } \\
\text { treatment }\end{array}$ & $\begin{array}{l}\text { Identification of } \\
\text { participants }\end{array}$ & $\begin{array}{l}\text { Quantitative } \\
\text { descriptive }\end{array}$ & $440 / 178 / 59$ & $\begin{array}{l}\text { Various mass } \\
\text { mailing, media, } \\
\text { health service } \\
\text { referral and } \\
\text { community } \\
\text { outreach } \\
\text { strategies. }\end{array}$ & $\begin{array}{l}\text { Mass mailing and } \\
\text { health service } \\
\text { referral strategies } \\
\text { were moderately } \\
\text { effective. } \\
\text { Recruitment } \\
\text { uptake was } \\
\text { highest in } \\
\text { participants } \\
\text { identified through } \\
\text { health service } \\
\text { referral. }\end{array}$ & Fair \\
\hline
\end{tabular}


Table 1 Continued

\begin{tabular}{|c|c|c|c|c|c|c|c|c|}
\hline Author, year & Host RCT & $\begin{array}{l}\text { Host RCT } \\
\text { therapeutic area }\end{array}$ & $\begin{array}{l}\text { Recruitment } \\
\text { stage studied }\end{array}$ & $\begin{array}{l}\text { Recruitment } \\
\text { study design }\end{array}$ & $\begin{array}{l}\text { Screened/eligible } \\
\text { /randomised* }(\mathrm{n})\end{array}$ & Intervention(s) & $\begin{array}{l}\text { Summary of } \\
\text { findings }\end{array}$ & $\begin{array}{l}\text { Quality } \\
\text { assessment } †\end{array}$ \\
\hline $\begin{array}{l}\text { Kumar et al, } \\
2012^{43}\end{array}$ & Not specified & $\begin{array}{l}\text { Prostate cancer } \\
\text { prevention }\end{array}$ & $\begin{array}{l}\text { Identification of } \\
\text { participants }\end{array}$ & $\begin{array}{l}\text { Quantitative } \\
\text { descriptive }\end{array}$ & $3547 / 167 / 74$ & $\begin{array}{l}\text { Various media, } \\
\text { health service } \\
\text { referral and } \\
\text { community } \\
\text { outreach } \\
\text { strategies. }\end{array}$ & $\begin{array}{l}\text { Principal } \\
\text { investigator } \\
\text { referral was the } \\
\text { only effective } \\
\text { recruitment } \\
\text { strategy. TV, } \\
\text { newspaper, print } \\
\text { and web-based } \\
\text { communications } \\
\text { and distribution } \\
\text { of posters and } \\
\text { flyers resulted } \\
\text { in very few } \\
\text { screenings. }\end{array}$ & Poor \\
\hline
\end{tabular}

$\begin{array}{lll}\begin{array}{l}\text { Kusek et al, } \\ 2002^{44}\end{array} & \text { MTOPS } & \begin{array}{l}\text { B } \\ \text { try }\end{array} \\ \text { Lee et al, } 2011^{45} & \text { CAMUS } & \text { B }\end{array}$

Benign prostatic Identification of Quantitative

$4170 / N R / 2931$

Various mass participants descriptive mailing, media, health service referral and community outreach strategies.

Newspaper advertising and stories, and mass mailings were the most effective recruitment strategies. Benign prostatic Identification of Quantitative 1032/NR/369 Various mass Newspaper, hyperplasia participants descriptive mailing, media, radio and online health service advertising, referral and and mass community mailing were the

outreach most effective strategies.

recruitment strategies.

Emailing was less effective than traditional mailing.

\begin{tabular}{|c|c|c|c|c|c|c|c|c|}
\hline $\begin{array}{l}\text { Moinpour et al, } \\
2000^{46}\end{array}$ & PCPT & $\begin{array}{l}\text { Prostate cancer } \\
\text { prevention }\end{array}$ & $\begin{array}{l}\text { Identification of } \\
\text { participants }\end{array}$ & Before and after & NR/NR/18,822‡ & $\begin{array}{l}\text { Site-directed } \\
\text { minority-targeted } \\
\text { recruitment } \\
\text { strategies } \\
\text { conducted by } \\
\text { funded minority } \\
\text { recruiter site staff. }\end{array}$ & $\begin{array}{l}\text { Minority-targeted } \\
\text { recruitment } \\
\text { strategies were } \\
\text { not effective at } \\
\text { four of the five } \\
\text { sites awarded } \\
\text { funds for a } \\
\text { minority recruiter. }\end{array}$ & Poor \\
\hline $\begin{array}{l}\text { Donovan et al, } \\
2002^{35}\end{array}$ & $\begin{array}{l}\text { PROTECT } \\
\text { (feasibility) }\end{array}$ & $\begin{array}{l}\text { Prostate cancer } \\
\text { treatment }\end{array}$ & $\begin{array}{l}\text { Participant } \\
\text { information and } \\
\text { consent }\end{array}$ & Before and after & NR/155/108 & $\begin{array}{l}\text { Site training } \\
\text { and guidance } \\
\text { documents } \\
\text { to address } \\
\text { recruitment } \\
\text { issues identified } \\
\text { through } \\
\text { qualitative } \\
\text { research. }\end{array}$ & $\begin{array}{l}\text { Recruitment rates } \\
\text { increased after } \\
\text { introduction of } \\
\text { the recruitment- } \\
\text { focused site } \\
\text { training and } \\
\text { guidance. }\end{array}$ & Fair \\
\hline $\begin{array}{l}\text { Donovan et al, } \\
2003^{49}\end{array}$ & $\begin{array}{l}\text { PROTECT } \\
\text { (feasibility) }\end{array}$ & $\begin{array}{l}\text { Prostate cancer } \\
\text { treatment }\end{array}$ & $\begin{array}{l}\text { Participant } \\
\text { information and } \\
\text { consent }\end{array}$ & $\mathrm{RCT}$ & NR/167/103 & $\begin{array}{l}\text { Recruitment } \\
\text { visit conducted } \\
\text { by nurse vs } \\
\text { recruitment visit } \\
\text { conducted by } \\
\text { urologist. }\end{array}$ & $\begin{array}{l}\text { Recruitment rates } \\
\text { in the urologist } \\
\text { and the nurse } \\
\text { groups were } \\
\text { not significantly } \\
\text { different. } \\
\text { Recruitment by } \\
\text { nurse was more } \\
\text { cost-effective } \\
\text { than recruitment } \\
\text { by urologist. }\end{array}$ & Good \\
\hline $\begin{array}{l}\text { Donovan et al, } \\
2009^{36}\end{array}$ & PROTECT & $\begin{array}{l}\text { Prostate cancer } \\
\text { treatment }\end{array}$ & $\begin{array}{l}\text { Participant } \\
\text { information and } \\
\text { consent }\end{array}$ & Before and after & NR/2664/1643‡ & $\begin{array}{l}\text { Site training } \\
\text { and guidance } \\
\text { documents } \\
\text { to address } \\
\text { recruitment } \\
\text { issues identified } \\
\text { through } \\
\text { qualitative } \\
\text { research. }\end{array}$ & $\begin{array}{l}\text { Recruitment rates } \\
\text { fell slightly after } \\
\text { introduction of } \\
\text { the recruitment- } \\
\text { focused site } \\
\text { training and } \\
\text { guidance. }\end{array}$ & Fair \\
\hline
\end{tabular}


Table 1 Continued

\begin{tabular}{|c|c|c|c|c|c|c|c|c|}
\hline Author, year & Host RCT & $\begin{array}{l}\text { Host RCT } \\
\text { therapeutic area }\end{array}$ & $\begin{array}{l}\text { Recruitment } \\
\text { stage studied }\end{array}$ & $\begin{array}{l}\text { Recruitment } \\
\text { study design }\end{array}$ & $\begin{array}{l}\text { Screened/eligible } \\
\text { /randomised* (n) }\end{array}$ & Intervention(s) & $\begin{array}{l}\text { Summary of } \\
\text { findings }\end{array}$ & $\begin{array}{l}\text { Quality } \\
\text { assessment } †\end{array}$ \\
\hline $\begin{array}{l}\text { Eccles et al, } \\
2013^{34}\end{array}$ & $\begin{array}{l}\text { SABRE } 1 \\
\text { (feasibility) }\end{array}$ & $\begin{array}{l}\text { Prostate cancer } \\
\text { treatment }\end{array}$ & $\begin{array}{l}\text { Participant } \\
\text { information and } \\
\text { consent }\end{array}$ & $\mathrm{RCT}$ & $286 / 30 / 4$ & $\begin{array}{l}30 \text { min decision } \\
\text { aid video } \\
\text { providing trial } \\
\text { information vs } \\
\text { control (standard } \\
\text { information). }\end{array}$ & $\begin{array}{l}\text { Too few } \\
\text { participants } \\
\text { were recruited } \\
\text { to assess } \\
\text { effectiveness } \\
\text { of the decision } \\
\text { aid video. Some } \\
\text { indication that the } \\
\text { video may have } \\
\text { decreased the } \\
\text { recruitment rate } \\
\text { when compared } \\
\text { with control. }\end{array}$ & Fair \\
\hline $\begin{array}{l}\text { Wallace et al, } \\
2006^{50}\end{array}$ & SPIRIT & $\begin{array}{l}\text { Prostate cancer } \\
\text { treatment }\end{array}$ & $\begin{array}{l}\text { Participant } \\
\text { information and } \\
\text { consent }\end{array}$ & Before and after & NR/290/32 & $\begin{array}{l}\text { Multidisciplinary } \\
\text { group information } \\
\text { session prior } \\
\text { to recruitment } \\
\text { vs one-on-one } \\
\text { recruitment visit. }\end{array}$ & $\begin{array}{l}\text { Recruitment } \\
\text { rates increased } \\
\text { after introduction } \\
\text { of the } \\
\text { multidisciplinary } \\
\text { group information } \\
\text { sessions. }\end{array}$ & Fair \\
\hline Ford et al, $2004^{51}$ & $\begin{array}{l}\text { PLCO/AAMEN } \\
\text { project }\end{array}$ & $\begin{array}{l}\text { Prostate, lung } \\
\text { and colorectal } \\
\text { cancer screening }\end{array}$ & $\begin{array}{l}\text { Identification } \\
\text { of participants, } \\
\text { assessment } \\
\text { of eligibility } \\
\text { and patient } \\
\text { information and } \\
\text { consent }\end{array}$ & $\mathrm{RCT}$ & 17 770/12 400/376 & $\begin{array}{l}\text { Three recruitment } \\
\text { approaches } \\
\text { of increasing } \\
\text { intensity targeted } \\
\text { at African- } \\
\text { American men, } \\
\text { compared } \\
\text { with standard } \\
\text { recruitment } \\
\text { approach. }\end{array}$ & $\begin{array}{l}\text { The most } \\
\text { intensive } \\
\text { approach to } \\
\text { screening, which } \\
\text { included face-to- } \\
\text { face screening } \\
\text { in a church } \\
\text { setting, resulted } \\
\text { in a higher } \\
\text { recruitment rate } \\
\text { than control. The } \\
\text { improvement } \\
\text { was statistically } \\
\text { significant but } \\
\text { small. Other } \\
\text { less intense } \\
\text { approaches were } \\
\text { no better than } \\
\text { control. }\end{array}$ & Fair \\
\hline $\begin{array}{l}\text { Lane et al, } \\
2011^{37}\end{array}$ & PROTECT & $\begin{array}{l}\text { Prostate cancer } \\
\text { treatment }\end{array}$ & $\begin{array}{l}\text { Assessment } \\
\text { of eligibility } \\
\text { and participant } \\
\text { information and } \\
\text { consent }\end{array}$ & Before and after & NR/2664/1643‡ & $\begin{array}{l}\text { Peer-conducted } \\
\text { site monitoring } \\
\text { visits. }\end{array}$ & $\begin{array}{l}\text { Recruitment } \\
\text { issues were } \\
\text { identified at } \\
\text { two out of eight } \\
\text { monitored } \\
\text { sites. Specific } \\
\text { recruitment } \\
\text { metrics (consent } \\
\text { form return rate, } \\
\text { reduction in } \\
\text { health-related } \\
\text { exclusions) } \\
\text { improved at } \\
\text { these two } \\
\text { sites following } \\
\text { monitoring. } \\
\text { The impact of } \\
\text { the monitoring } \\
\text { intervention } \\
\text { on overall } \\
\text { recruitment was } \\
\text { not reported. }\end{array}$ & Poor \\
\hline
\end{tabular}

${ }^{*}$ Refers to number of participants screened (including prescreening), eligible (approached for consent) and randomised to the host RCT as part of the recruitment study. †Quality rated as good, fair or poor with respect to the quantitative recruitment-related outcomes of interest in this systematic review.

‡Study did not report number of participants included in the recruitment evaluation. Instead total numbers of participants in host RCT are reported.

AAMEN, African-American Men; CAMUS, Complementary and Alternative Medicines Trial for Urological Symptoms; EASE, Eating, Activity , and Stress Education; MTOPS, Medical Therapy of Prostatic Symptom; NR, not reported; PLCO, Prostate, Lung, Colorectal and Ovarian Cancer Screening Trial; PCPT, Prostate Cancer Prevention Trial; PROTECT, Prostate Testing for Cancer and Treatment; RCT, randomised controlled trial; SABRE, Surgery Against Brachytherapy-a Randomised Evaluation; SELECT, Selenium and Vitamin E Cancer Prevention Trial; SPIRIT, Surgical Prostatectomy versus Interstitial Radiation Intervention Trial; T, Testosterone TV, television.

patient databases, commercial mailing lists, volunteer databases and lists of physicians and university employees.

Excluding poor studies, mailing referrals contributed $18 \%-100 \%$ of enrolled participants in the studies that used mailings. ${ }^{38} 40424445$ Uptake was very low across all studies $(0.09 \%-1.0 \%$ of mail recipients went on to be randomised to the host RCT). ${ }^{38} 404245$ The direct cost of mailings ranged from $\$ 59$ to $\$ 259$ per participant 


\begin{tabular}{lc}
\hline \multicolumn{2}{l}{ Table 2 Summary characteristics of included studies } \\
\hline Description & No of studies \\
\hline Therapeutic area of host RCT & 11 \\
Cancer - prostate & 2 \\
\hline Benign prostatic hyperplasia & 1 \\
\hline Testosterone & 1 \\
\hline Suicide & 1 \\
\hline Cancer-various & \\
Host RCT type & 10 \\
\hline Treatment & 5 \\
\hline Prevention & 1 \\
\hline Screening & \\
Recruitment study design & 10 \\
\hline Quantitative descriptive & 3 \\
\hline Randomised controlled trial & 2 \\
\hline Before and after study & 1 \\
\hline Non-randomised controlled study & \\
\hline No of study participants in recruitment & \\
study & 6 \\
\hline 0-999 & 5 \\
\hline 1000-4999 & 2 \\
\hline $5000-9999$ & 3 \\
\hline 10 000+ & 16 \\
\hline TOTAL recruitment studies included & \\
\hline
\end{tabular}

$\mathrm{RCT}$, randomised controlled trial.

enrolled. ${ }^{3840}$ In one study, postal invitations had a higher uptake than email invitations $(0.4 \%$ of mail recipients enrolled vs $0.1 \%$ of email recipients).$^{45}$ However, mail and email lists were drawn from dissimilar populations making a direct, unadjusted comparison problematic.

In one study, ${ }^{40}$ mailing women who were past research participants and asking them to invite their spouses resulted in higher recruitment uptake $(4.3 \%$ vs $1.0 \%$ enrolled) and lower cost per participant ( $\$ 59$ per enrolment vs \$259 per enrolment) compared with mailing men on a home owners database. However, the home owners mailing list was much larger than the past-participant mailing list (60000 vs 800 members), and so $95 \%$ of participants were recruited through the home owners mailing list despite the lower uptake rate. ${ }^{40}$

Media coverage and advertising

Six studies ${ }^{39} 41-45$ described a variety of media strategies including news stories on television ${ }^{44}$ and in newspapers ${ }^{42}$; advertising on television, ${ }^{39434}$ radio $^{394445}$ and in newspapers ${ }^{39} 43-45$; listing the study on the clinicaltrials. gov website ${ }^{39} 43$; other online advertising ${ }^{45}$; and inclusion in military retiree and medical institution newsletters. ${ }^{44}$

Two studies reported that media strategies were effective, accounting for $35 \%{ }^{45}$ and $54 \%^{44}$ of enrolments. The remaining four studies were excluded for poor

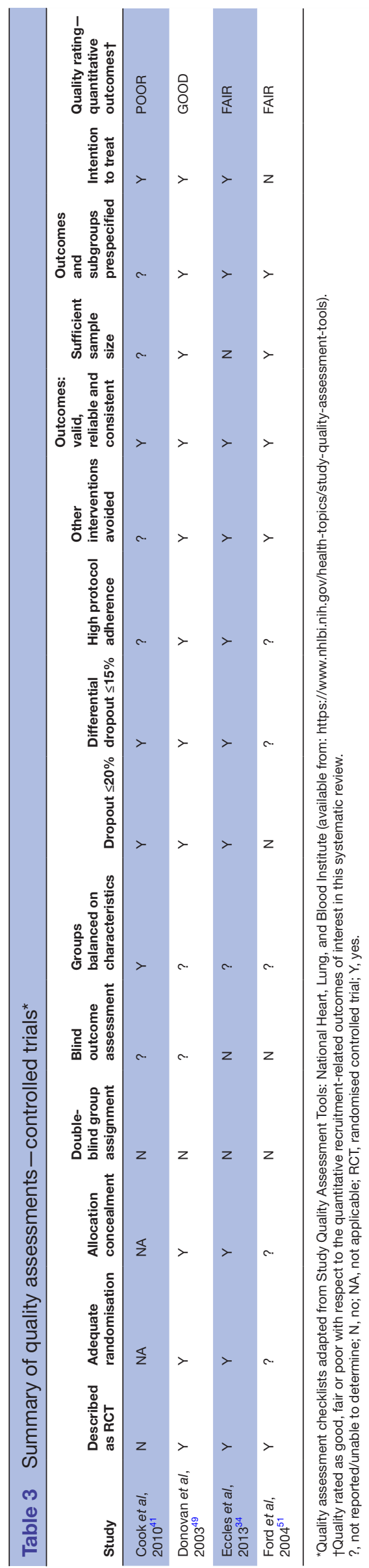



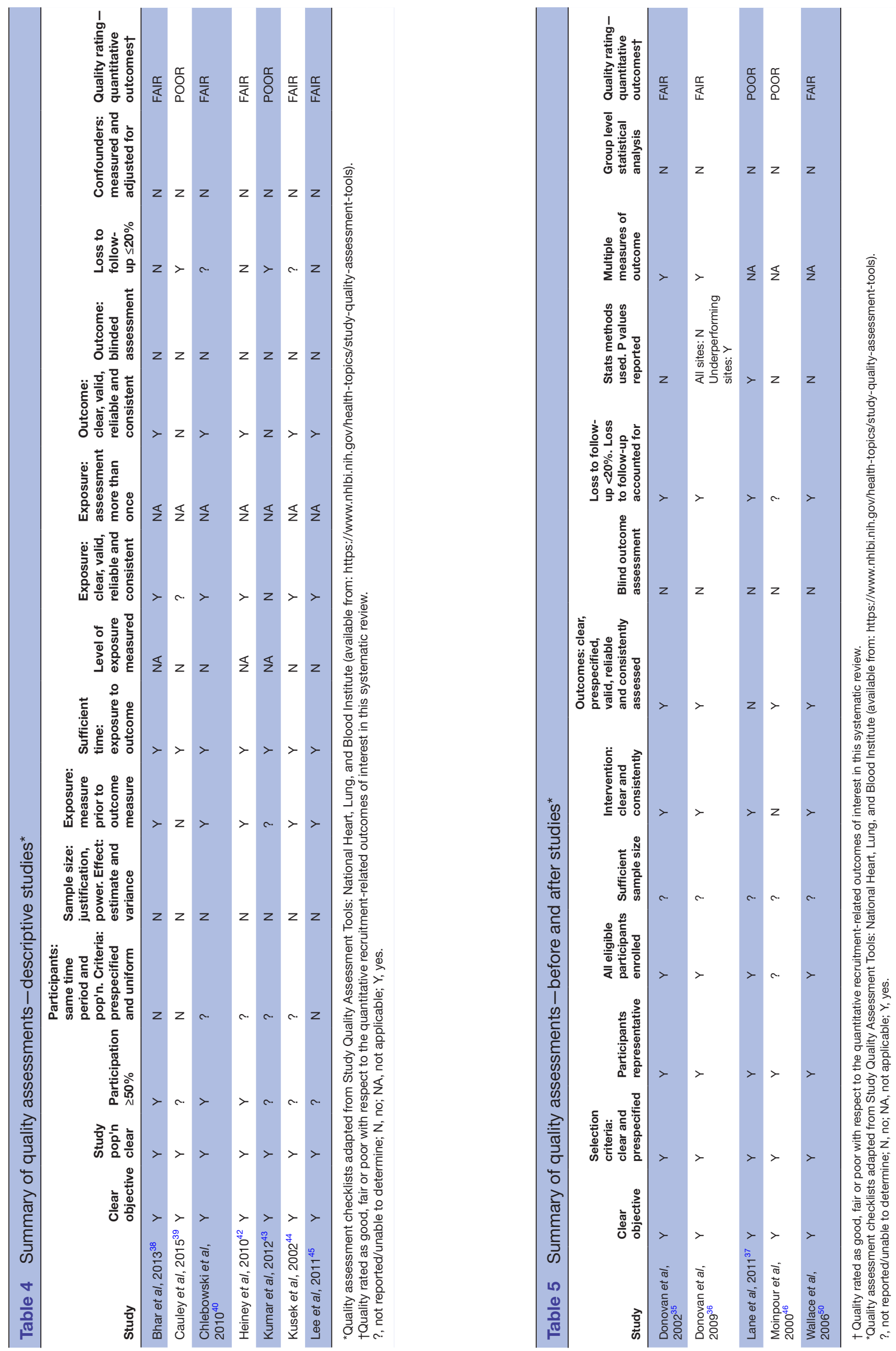

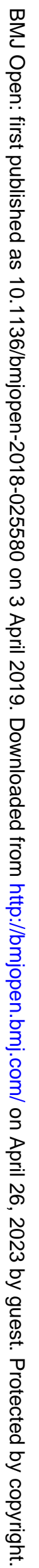


Table 6 Strategy uptake in included studies

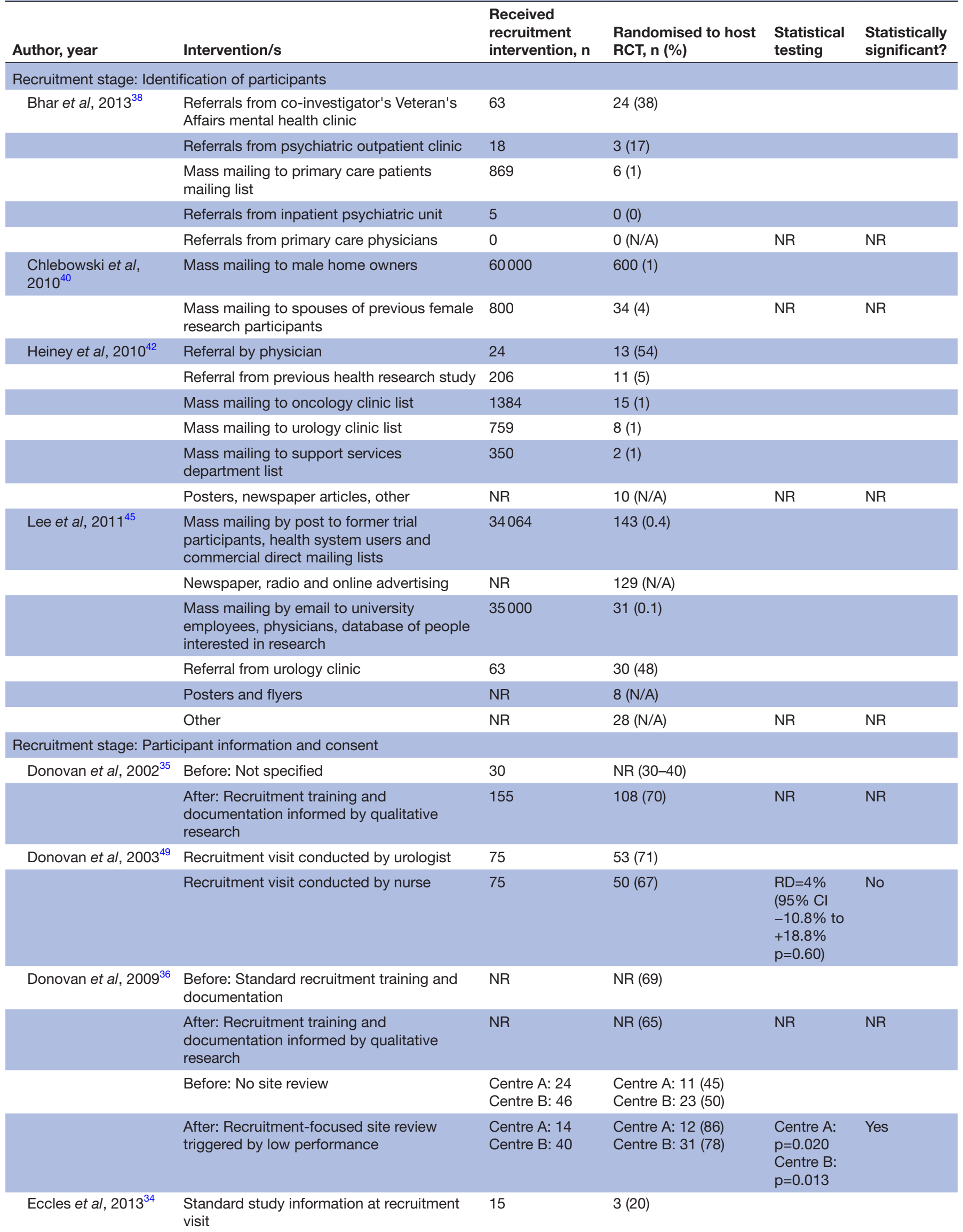




\begin{tabular}{|c|c|c|c|c|c|}
\hline Author, year & Intervention/s & $\begin{array}{l}\text { Received } \\
\text { recruitment } \\
\text { intervention, } \mathrm{n}\end{array}$ & $\begin{array}{l}\text { Randomised to host } \\
\text { RCT, n (\%) }\end{array}$ & $\begin{array}{l}\text { Statistical } \\
\text { testing }\end{array}$ & $\begin{array}{l}\text { Statistically } \\
\text { significant? }\end{array}$ \\
\hline & Decision aid video at recruitment visit & 15 & $1(7)$ & NR & NR \\
\hline Wallace et al, $2006^{50}$ & $\begin{array}{l}\text { After: Multidisciplinary group information } \\
\text { session }\end{array}$ & 263 & $32(12)$ & NR & NR \\
\hline \multirow{2}{*}{ Ford et al, $2004^{51}$} & $\begin{array}{l}\text { Arm B: Enhanced mailed invitation, } \\
\text { telephone screening by African-American } \\
\text { interviewer, collection of baseline data by } \\
\text { phone }\end{array}$ & 3075 & $87(3)$ & & \\
\hline & $\begin{array}{l}\text { Arm D (control): Standard mailed invitation, } \\
\text { telephone screening by African-American or } \\
\text { Caucasian interviewer, collection of baseline } \\
\text { data by mail }\end{array}$ & 3297 & $95(3)$ & $\begin{array}{l}\text { Difference } \\
\text { between } \\
\text { arms B, } \\
\mathrm{C} \text { and D: } \\
\mathrm{p}=0.66\end{array}$ & No \\
\hline
\end{tabular}

*Strategy uptake defined as the percentage of people receiving the recruitment intervention who went on to be randomised to the host RCT. Studies that did not report the number of participants receiving the recruitment intervention excluded. Poor quality studies excluded. NR , not reported; RCT, randomised controlled trial; N/A, not applicable.

quality or lack of media-related outcome reporting. One study ${ }^{44}$ reported that newspapers were the largest source of recruited participants $(30 \%)$ followed by radio (9\%), newsletters (8\%) and television (7\%). Although five studies mentioned using paid advertising only one poor quality study ${ }^{39}$ reported costs with television being the cheapest ( $\$ 46$ per screening), followed by radio advertising (\$51 per screening) and print most expensive (\$105 per screening). All were more expensive than mass mailing (\$38 per screening).

\section{Health service referral}

Health service referral was defined as identification of prospective participants by a health service provider. Only strategies which involved the health service provider having performed some initial screening were included. Where mass mail outs were performed using clinic lists without prior clinical screening, these were categorised as a mass mailing. Five studies ${ }^{38-45}$ sought referrals from a variety of sources including outpatient clinics and medical centres, physicians (both site investigators and community physicians), hospital inpatient lists and lists of previous prostate cancer research participants.

In studies of fair quality, the health service referral sources fell into two broad categories; those that were affiliated with a study site (ie, referrals through an existing clinical pathway or a study investigator's clinic) and those that were not. For studies that could draw referrals from affiliated health services, ${ }^{38} 42$ health service referral was the most effective participant referral strategy contributing $41 \%{ }^{42}$ and $82 \%{ }^{38}$ of participants. For the remaining two studies, which sought referrals from health services not linked to the study, health services referrals were comparatively ineffective, contributing only $8 \%$ and $10 \%^{44}$ of participants.

Recruitment uptake from health services referral was generally higher than other strategies but was highly variable, ranging from $0 \%$ to $54 \%$ of referrals being randomised to the host RCT. ${ }^{3842} 45$ Only one study ${ }^{38}$ reported cost-effectiveness. Referrals from a variety of health services cost $\$ 101$ per participant randomised on average (see table 8 for details). Referral from an affiliated health service was the cheapest referral source ( $\$ 44$ per participant randomised). ${ }^{38}$

\section{Community outreach}

Seven studies evaluated community outreach strategies $^{3941-46}$ including posters displayed in community locations and healthcare clinics, and presentations to health service providers and the public.

Two studies reported that community outreach activities were ineffective, accounting for only $2 \%{ }^{45}$ and $4 \%{ }^{44}$ of participants. The remaining five studies were excluded due to poor quality or failure to report the outcome of community outreach activities. 
Table 7 Contribution of participant identification strategies to recruitment ${ }^{\star}$

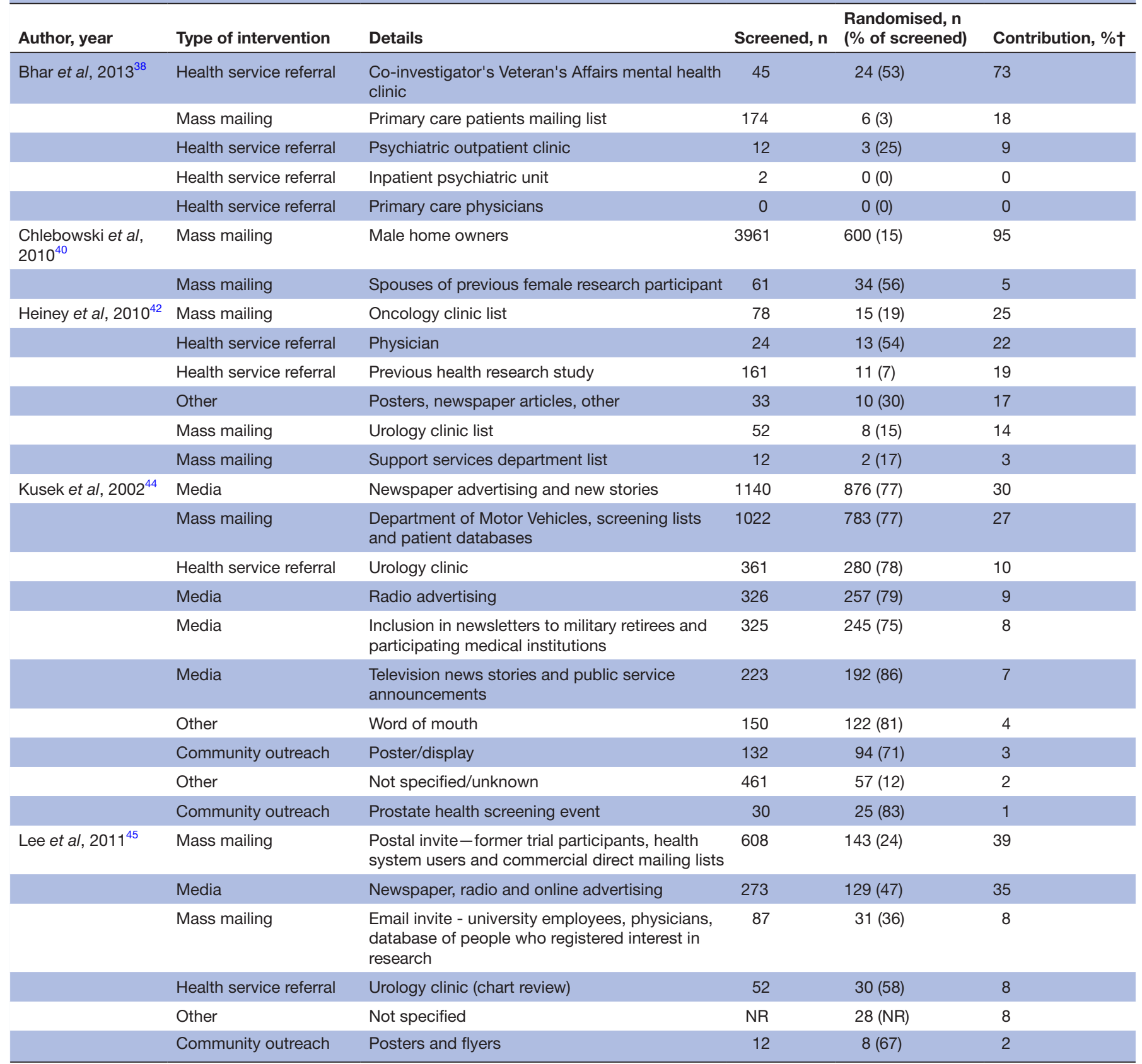

*Poor quality studies excluded.

tContribution defined as the percentage of all participants randomised to the host randomised controlled trial who were randomised as a result of a particular recruitment strategy.

NR, not reported.

\section{Patient information and consent}

Five studies evaluated strategies to improve the patient information and consent process. ${ }^{34-36} 4950$ The strategy uptake reported in each of these studies is shown in table 6 . The studies aimed to improve either the content of the information provided to the participant at the recruitment visit or the mechanism by which that information was provided. All studies in this category were hosted within prostate cancer treatment trials, perhaps reflecting the challenges inherent in recruiting participants to prostate cancer trials where treatment options may be diverse, for example, surgery, radiation and watchful waiting.

Two papers evaluated a trial-specific recruitment training intervention delivered to site-based recruitment staff and implemented within the feasibility and main phases of the PROTECT trial. ${ }^{35} 36$ The intervention involved audio-taping recruitment interviews and performing qualitative to investigate how trial information was delivered to participants at the recruitment visit and how this may impact consent rates. Results of this 
Table 8 Cost of recruitment strategies*

\begin{tabular}{|c|c|c|c|c|c|c|}
\hline Author, year & Costs reported & $\begin{array}{l}\text { Recruitment } \\
\text { phase }\end{array}$ & Intervention/s & $\begin{array}{l}\text { Randomised, } \\
\mathrm{n}\end{array}$ & Cost & $\begin{array}{l}\text { Cost per } \\
\text { participant }\end{array}$ \\
\hline \multirow[t]{3}{*}{ Bhar et al, $2013^{38}$} & $\begin{array}{l}\text { Direct cost } \\
\text { (stationary, postage, } \\
\text { phone calls and } \\
\text { catering) and } \\
\text { indirect cost (staff } \\
\text { time) }\end{array}$ & $\begin{array}{l}\text { Identification of } \\
\text { participants }\end{array}$ & $\begin{array}{l}\text { Mass mailing-primary } \\
\text { care patients mailing list }\end{array}$ & 6 & US\$3813 & US\$636 \\
\hline & & & $\begin{array}{l}\text { Health services referral- } \\
\text { psychiatric outpatient clinic }\end{array}$ & 3 & US $\$ 497$ & US\$166 \\
\hline & & & $\begin{array}{l}\text { Health services referral- } \\
\text { primary care physicians }\end{array}$ & 0 & US\$643 & N/A \\
\hline $\begin{array}{l}\text { Chlebowski et al, } \\
2010^{40}\end{array}$ & & & $\begin{array}{l}\text { Mass mailing-spouses of } \\
\text { previous female participant }\end{array}$ & 34 & US\$2000 & US\$59 \\
\hline \multirow[t]{2}{*}{$\begin{array}{l}\text { Donovan et al, } \\
2003^{49}\end{array}$} & $\begin{array}{l}\text { Salary and on-costs } \\
\text { for staff time }\end{array}$ & $\begin{array}{l}\text { Participant } \\
\text { information and } \\
\text { consent }\end{array}$ & $\begin{array}{l}\text { Recruitment visit performed } \\
\text { by urologist }\end{array}$ & 53 & NR & $£ 43.29$ \\
\hline & & & $\begin{array}{l}\text { Recruitment visit performed } \\
\text { by nurse }\end{array}$ & 50 & NR & $£ 36.40$ \\
\hline
\end{tabular}

${ }^{*}$ Poor quality studies excluded.

$\mathrm{NR}$, not reported.

analysis then guided the development of the training intervention. After the implementation of the intervention, the recruitment rate was observed to increase from $30 \%-40 \%$ to $70 \%$ during the feasibility stage and to remain between $69 \%$ and $65 \%$ during the main study. An evaluation of a secondary, intensive training process for underperforming sites found that recruitment rates increased from $45 \%$ to $86 \%(\mathrm{p}=0.020)$ at one site and from $50 \%$ to $78 \%(\mathrm{p}=0.013)$ at another site but numbers at these two sites were small. ${ }^{36}$
Other participant information and consent interventions were evaluated in one study each. Recruitment by nurses was found to be more cost-effective than recruitment by urologists ( $£ 36.40$ vs $£ 43.29$ per screening) and resulted in similar rates of consent $(67 \% \text { vs } 71 \% \mathrm{p}=0.60 .)^{49}$ Multidisciplinary, group information sessions increased the consent rate from $0 \%$ to $16 \%$ when compared with a one-on-one recruitment consultation. ${ }^{50} \mathrm{~A} 30 \mathrm{~min}$ decision aid video presented at the recruitment visit may have reduced the consent rate in one study although the study was underpowered. ${ }^{34}$

Table 9 A summary of the contribution of participant identifications strategies to randomised controlled trial recruitment ${ }^{\star} \dagger$

\begin{tabular}{llllllr}
\hline & & $\begin{array}{l}\text { Media } \\
\text { coverage and } \\
\text { advertising }\end{array}$ & $\begin{array}{l}\text { Health service } \\
\text { referrals }\end{array}$ & $\begin{array}{l}\text { Community } \\
\text { outreach }\end{array}$ & $\begin{array}{l}\text { Other, } \\
\text { unspecified, } \\
\text { unknown }\end{array}$ & $\begin{array}{l}\text { Participants } \\
\text { enrolled }\end{array}$ \\
\hline Kusek, $2002^{44}$ & $783(27 \%)$ & $1570(54 \%)$ & $280(10 \%)$ & $119(4 \%)$ & $179(6 \%)$ & $2931(100 \%)$ \\
$\begin{array}{l}\text { Chlebowski, } \\
2010^{40}\end{array}$ & $634(100 \%)$ & - & - & - & - & $634(100 \%)$ \\
Lee, $2011^{45}$ & $174(47 \%)$ & $129(35 \%)$ & $30(8 \%)$ & $8(2 \%)$ & $28(8 \%)$ & $369(100 \%)$ \\
Heiney, $2010^{42}$ & $25(42 \%)$ & NR & $24(41 \%)$ & NR & - & $59(100 \%)$ \\
Bhar, $2013^{38}$ & $6(18 \%)$ & - & $27(82 \%)$ & - & - & $33(100 \%)$ \\
\hline
\end{tabular}

${ }^{*}$ Contribution defined as the number of participants randomised as a result of each strategy (percentage of all participants randomised). †Poor quality studies excluded.

NR, not reported separately. In total, media and community strategies accounted for $17 \%$ of enrolled participants in this study. 


\section{Strategies addressing multiple stages of the recruitment} process

Two studies evaluated strategies that addressed the recruitment process as a whole (identification of participants, assessment of eligibility, provision of participant information and seeking of consent) rather than one specific stage of recruitment. One study evaluated the impact of peer-conducted site monitoring visits on recruitment. ${ }^{37}$ The study reported that of the eight sites visited monitoring identified specific recruitment process issues at two sites. After monitoring, one site altered their process for participant reminders and subsequently consent form return rates increased by $5 \%$. At another site, monitoring uncovered that eligibility criteria were being incorrectly applied and subsequently incorrect exclusion of prospective participants decreased by $5 \%$. While these improvements to site processes are likely to have improved recruitment, the study did not report the impact on overall recruitment.

Another study evaluated four different approaches to recruit African-American men to a cancer screening trial. ${ }^{51}$ The study found that the most intensive intervention (mailing invitation endorsed by African-American community leader, phone screening by African-American interviewer and gathering baseline information at a church-based group session with transport provided) increased recruitment uptake from $2.9 \%$ to $3.9 \%$ compared with control (standard mailed invitation, phone screening by African-American or non-African-American interviewer and collection of baseline forms by mail). While this difference was statistically significant $(\mathrm{p}<0.01)$, it was small in absolute magnitude, and the cost of the most intensive intervention is likely to have been high although cost data were not reported. Other approaches that included less intensive combinations of mailing invitation endorsed by an African-American community leader, phone screening by African-American interviewer and gathering baseline information by phone did not result in a statistically significant increase in recruitment uptake compared with control.

\section{DISCUSSION}

\section{Principal findings}

In this review, we aimed to evaluate recruitment strategies in RCTs of men aged 50 years and older. We found that the best approaches for identifying participants were referral through an affiliated health service provider, media coverage and mass mailings. Community outreach activities and referrals from unaffiliated health service providers were not effective strategies for improving recruitment. Recruitment was improved by trial-specific training informed by qualitative analysis of the recruitment visit and delivered to site-based recruitment staff.

\section{Context within the existing literature}

This review included only recruitment evaluations in RCTs of men aged 50 years and over and was dominated by RCTs in prostate cancer. However, our findings were broadly consistent with recruitment studies in both men and women, ranging in age from young adults ${ }^{14}$ to the elderly, ${ }^{12}$ and across primary care ${ }^{52}$ disease prevention, ${ }^{25}$ health screening, ${ }^{53}{ }^{54}$ and cancer and surgical ${ }^{55}$ research. Nonetheless, some previous studies have reported differences in strategy effectiveness based on age and gender. Mass mailing strategies were more effective in men than women, and effectiveness increased with age. ${ }^{25}$ By contrast, online advertising strategies were more effective in women than men, ${ }^{29} 56$ and most recruited participants were adolescents and young adults. ${ }^{28}$ Community outreach activities appeared to have limited effectiveness in the general population, ${ }^{26} 3057$ with some suggestion that they were more effective in women than in men. ${ }^{48}$ Elsewhere, two reviews reported that community outreach activities might be effective when recruiting hard-to-reach participants such as vulnerable ${ }^{58}$ and elderly ${ }^{12}$ populations. These reviews reported community outreach activities tailored to the specific target populations. Some tailoring was evident in the studies included in the current review (for example, holding screening sessions at men's health events ${ }^{43} 44$ and producing brochures in colours expected to appeal to men $^{42}$ ) but the context and content of community outreach activities were not described in detail. It is unknown whether further tailoring could have improved the effectiveness of community outreach activities in the recruitment of men aged 50 years and over. An upcoming Cochrane review may elucidate how age and gender modify the effect of specific recruitment strategies. ${ }^{59}$

\section{Strengths and limitations}

This review is strengthened by the adaption of the SEAR framework to categorise the included studies. Research into recruitment strategies is fragmented, ${ }^{5}$ and researchers seeking evidence-based solutions to their recruitment challenges may find the current evidence difficult to digest. Categorising studies according to the stage of the recruitment process rather than categorising by intervention characteristics has a number of advantages. First, it is intuitive to use and understand since it mirrors real-world trial processes. Second, for researchers using the SEAR framework to collect recruitment data and identify recruitment challenges, our review provides a roadmap for navigating the available evidence and selecting the most promising interventions to address these challenges.

By grouping studies according to the SEAR framework, our review uncovered inconsistencies in how strategies to identify prospective participants were evaluated. All studies in this category reported strategy contributions to overall recruitment but only four studies reported strategy uptake and two reported strategy costs. There was a lack of consensus across studies on which of these outcomes was most appropriate and it was unclear how studies decided whether strategies were effective or not. Intuitively, these three possible outcomes (contribution, 
uptake and cost) are, individually, insufficient to evaluate overall strategy effectiveness. For example, if a strategy contributed $80 \%$ of study participants does this indicate that the strategy was effective or simply that few other strategies were used? Likewise, if a strategy was low cost but resulted in few participants being randomised was it more or less effective than an expensive strategy that delivered large numbers of participants? Greater transparency in how strategies to identify participants are selected and assessed, and the costs involved would assist with the interpretation of study results in this area.

Of the estimated large number of men's health RCTs conducted worldwide since 2000, only 16 studies of recruitment strategy evaluation were found, and 12 of these studies were related to prostate cancer (four from a single prostate cancer trial). Consequently, this review likely describes only a small fraction of the recruitment practices used to recruit men aged 50 years and older to RCTs and may be subject to publication bias. We included only single-gender, men's RCTs in order to focus on gender-specific recruitment strategies. Several included studies described recruitment strategies that appeared to be male-focused (identifying participants through veteran's groups and health services, ${ }^{39} 44$ holding screening sessions at men's health events, ${ }^{43} 44$ offering screening outside normal working hours ${ }^{41}$ and producing brochures in colours expected to appeal to $\mathrm{men}^{42}$ ). However, no study explicitly presented a gender-sensitised approach to recruitment or addressed the literature on men's health preferences. ${ }^{60}$ Since studies evaluating recruitment to RCTs of both men and women were excluded, the results presented in this review are likely to be most relevant to the small but growing number of RCTs in men only. ${ }^{61}$ However, our approach to synthesising recruitment evidence and evaluating strategy effectiveness by recruitment stage may be relevant to recruitment to RCTs more broadly.

This review considered only quantitative evidence from recruitment evaluation studies, a common approach in systematic reviews of recruitment strategies ${ }^{781314}$ However, qualitative research methods also have the potential to address recruitment challenges ${ }^{62}$ and several included studies presented both quantitative and qualitative evidence. Future systematic reviews of recruitment strategies may be strengthened by synthesising all available evidence using a mixed methods approach. ${ }^{63}$

We recommend caution when implementing recruitment strategies based on our findings since generalisability is hampered by weak recruitment study design, and insufficient reporting of the intervention content, context, delivery and cost in many of the included studies. Based on recently proposed criteria ${ }^{64}$ additional evaluations of all potentially effective strategies identified in this review are likely to be of merit.

\section{Implications for research}

Our review uncovered areas of uncertainty across all stages of the recruitment process. In particular, further research is needed to assess whether gender-sensitised strategies can enhance recruitment of men aged 50 years and over to RCTs, and to assess the effectiveness of online advertising and promotions to recruit this demographic group. Future research may benefit from being conducted as a prospectively designed Study Within a Trial, following the recent guidance provided by Trial Forge. ${ }^{65}$ Researchers are encouraged to reveal how strategy effectiveness was assessed and to report cost outcomes. Since there are many uncertainties in recruitment methods, research should address one or more of the priority recruitment questions recently identified by the Prioritising Recruitment in Randomised Trials study. ${ }^{66}$ This will not only improve the impact of individual studies but also deepen the body of recruitment evidence in general. ${ }^{8}$

Acknowledgements We thank Rod Dyson and Sherilyn Goldstone for their contributions to this work.

Contributors The review was conceived by KB and GW. KB performed the database searches. KB and GW performed eligibility checking. KB extracted the data from included studies and $K B$ and $L A$ performed the quality assessments. $K B$ wrote the first draft of the manuscript. KB, LA, AK, WH and GW reviewed and refined the manuscript and approved the final manuscript.

Funding KB is supported by a postgraduate scholarship from the NHMRC Clinical Trials Centre, University of Sydney.

Competing interests None declared.

Patient consent for publication Not required.

Provenance and peer review Not commissioned; externally peer reviewed.

Data sharing statement Extracted, summary, recruitment data are available on request from the corresponding author (karen.bracken@ctc.usyd.edu.au).

Open access This is an open access article distributed in accordance with the Creative Commons Attribution Non Commercial (CC BY-NC 4.0) license, which permits others to distribute, remix, adapt, build upon this work non-commercially, and license their derivative works on different terms, provided the original work is properly cited, appropriate credit is given, any changes made indicated, and the use is non-commercial. See: http://creativecommons.org/licenses/by-nc/4.0/.

\section{REFERENCES}

1. Walters SJ, Bonacho Dos Anjos Henriques-Cadby I, Bortolami $\mathrm{O}$, et al. Recruitment and retention of participants in randomised controlled trials: a review of trials funded and published by the United Kingdom Health Technology Assessment Programme. BMJ Open 2017;7:e015276.

2. Sully BG, Julious SA, Nicholl J. A reinvestigation of recruitment to randomised, controlled, multicenter trials: a review of trials funded by two UK funding agencies. Trials 2013;14:166.

3. McDonald AM, Knight RC, Campbell MK, et al. What influences recruitment to randomised controlled trials? A review of trials funded by two UK funding agencies. Trials 2006;7:9.

4. Tudur Smith C, Hickey H, Clarke M, et al. The trials methodological research agenda: results from a priority setting exercise. Trials 2014;15:32.

5. Bower P, Brueton V, Gamble C, et al. Interventions to improve recruitment and retention in clinical trials: a survey and workshop to assess current practice and future priorities. Trials 2014;15:399.

6. Treweek S, Lockhart P, Pitkethly M, et al. Methods to improve recruitment to randomised controlled trials: Cochrane systematic review and meta-analysis. BMJ Open 2013;3:e002360.

7. Caldwell PH, Hamilton S, Tan A, et al. Strategies for increasing recruitment to randomised controlled trials: systematic review. PLoS Med 2010;7:e1000368.

8. Treweek S, Pitkethly M, Cook J, et al. Strategies to improve recruitment to randomised trials. Cochrane Database Syst Rev 2018;2:MR000013.

9. Trivedi RB, Szarka JG, Beaver K, et al. Recruitment and retention rates in behavioral trials involving patients and a support person: a systematic review. Contemp Clin Trials 2013;36:307-18. 
10. Amon KL, Campbell AJ, Hawke C, et al. Facebook as a recruitment tool for adolescent health research: a systematic review. Acad Pediatr 2014;14:439-47.

11. Cooper C, Ketley D, Livingston G. Systematic review and metaanalysis to estimate potential recruitment to dementia intervention studies. Int J Geriatr Psychiatry 2014;29:515-25.

12. Provencher V, Mortenson WB, Tanguay-Garneau L, et al. Challenges and strategies pertaining to recruitment and retention of frail elderly in research studies: a systematic review. Arch Gerontol Geriatr 2014;59:18-24.

13. Boland J, Currow DC, Wilcock A, et al. A systematic review of strategies used to increase recruitment of people with cancer or organ failure into clinical trials: implications for palliative care research. J Pain Symptom Manage 2015;49:762-72.

14. Lam E, Partridge SR, Allman-Farinelli M. Strategies for successful recruitment of young adults to healthy lifestyle programmes for the prevention of weight gain: a systematic review. Obes Rev 2016;17:178-200.

15. Galli L, Knight R, Robertson S, et al. Using marketing theory to inform strategies for recruitment: a recruitment optimisation model and the txt2stop experience. Trials 2014;15:182.

16. Doyal L, Sex DL. Sex, gender, and health: the need for a new approach. BMJ 2001;323:1061-3.

17. Payne S. How can gender equity be addressed through health systems? Copenhagen: World Health Organization, 2009.

18. U.S. Food and Drug Administration. Guideline for the study and evaluation of gender differences in the clinical evaluation of drugs; notice. Fed Regist 1993;58:39406.

19. Coakley M, Fadiran EO, Parrish LJ, et al. Dialogues on diversifying clinical trials: successful strategies for engaging women and minorities in clinical trials. J Womens Health 2012;21:713-6.

20. Australian Burden of Disease Study. Impact and causes of illness and death in Australia: Australian Institute of Health and Welfare 2011 revised 2016 https://www.aihw.gov.au/getmedia/d4df9251-c4b6452f-a877-8370b6124219/19663.pdf.aspx?inline=true (accessed 23 March 2018).

21. The Health of Australian Males: Australian Institute of Health and Welfare. 2017 https://www.aihw.gov.au/reports/men-women/malehealth/contents/who-are (accessed 23 March 2018).

22. Macdonald JJ. Shifting paradigms: a social-determinants approach to solving problems in men's health policy and practice. Med J Aust 2006;185:456.

23. Smith JA, Robertson S. Men's health promotion: a new frontier in Australia and the UK? Health Promot Int 2008;23:283-9.

24. Hunt K, Wyke S, Gray CM, et al. A gender-sensitised weight loss and healthy living programme for overweight and obese men delivered by Scottish Premier League football clubs (FFIT): a pragmatic randomised controlled trial. The Lancet 2014;383:1211-21.

25. Rubin RR, Fujimoto WY, Marrero DG, et al. DPP Research Group. The Diabetes Prevention Program: recruitment methods and results. Control Clin Trials 2002;23:157-71.

26. Tate DF, LaRose JG, Griffin LP, et al. Recruitment of young adults into a randomized controlled trial of weight gain prevention: message development, methods, and cost. Trials 2014;15:326.

27. Pagoto SL, Schneider KL, Oleski JL, et al. Male Inclusion in Randomized Controlled Trials of Lifestyle Weight Loss Interventions. Obesity 2012;20:1234-9.

28. Whitaker C, Stevelink S, Fear N. The Use of Facebook in Recruiting Participants for Health Research Purposes: A Systematic Review. Med Internet Res 2017; 19:e290.

29. Huesch MD, Mukherjee D, Saunders EF. E-recruitment into a bipolar disorder trial using Facebook tailored advertising. Clin Trials 2018; 15:522-3.

30. Christensen T, Riis AH, Hatch EE, et al. Costs and Efficiency of Online and Offline Recruitment Methods: A Web-Based Cohort Study. J Med Internet Res 2017;19:e58.

31. Carter-Harris L, Bartlett Ellis R, Warrick A, et al. Beyond Traditional Newspaper Advertisement: Leveraging Facebook-Targeted Advertisement to Recruit Long-Term Smokers for Research. J Med Internet Res 2016;18:e117.

32. Wilson C, Rooshenas L, Paramasivan S, et al. Development of a framework to improve the process of recruitment to randomised controlled trials (RCTs): the SEAR (Screened, Eligible, Approached, Randomised) framework. Trials 2018;19:50.

33. Study Quality Assessment Tools: National Heart, Lung, and Blood Institute. https://www.nhlbi.nih.gov/health-topics/study-qualityassessment-tools (accessed 28 November 2017).

34. Eccles BK, Cross W, Rosario DJ, et al. SABRE 1 (Surgery Against Brachytherapy - a Randomised Evaluation): feasibility randomised controlled trial (RCT) of brachytherapy vs radical prostatectomy in low-intermediate risk clinically localised prostate cancer. BJU Int 2013;112:330-7.

35. Donovan J, Mills N, Smith M, et al. Improving design and conduct of randomised trials by embedding them in qualitative research: ProtecT (prostate testing for cancer and treatment) study. $\mathrm{Br} \mathrm{Med} J$ 2002;325:766-70.

36. Donovan JL, Athene Lane J, Peters TJ, et al. Development of a complex intervention improved randomization and informed consent in a randomized controlled trial. J Clin Epidemiol 2009;62:29-36.

37. Lane JA, Wade J, Down L, et al. A Peer Review Intervention for Monitoring and Evaluating sites (PRIME) that improved randomized controlled trial conduct and performance. $J$ Clin Epidemiol 2011;64:628-36.

38. Bhar SS, Wiltsey-Stirman S, Zembroski D, et al. Recruiting older men for geriatric suicide research. Int Psychogeriatr 2013;25:88-95.

39. Cauley JA, Fluharty L, Ellenberg SS, et al. Recruitment and Screening for the Testosterone Trials. J Gerontol A Biol Sci Med Sci 2015;70:1105-11.

40. Chlebowski RT, Menon R, Chaisanguanthum RM, et al. Prospective evaluation of two recruitment strategies for a randomized controlled cancer prevention trial. Clin Trials 2010;7:744-8.

41. Cook ED, Arnold KB, Hermos JA, et al. Impact of supplemental site grants to increase African American accrual for the Selenium and Vitamin E Cancer Prevention Trial. Clin Trials 2010;7:90-9.

42. Heiney SP, Arp Adams S, Drake BF, et al. Successful subject recruitment for a prostate cancer behavioral intervention trial. Clin Trials 2010;7:411-7.

43. Kumar N, Crocker T, Smith T, et al. Challenges and potential solutions to meeting accrual goals in a Phase II chemoprevention trial for prostate cancer. Contemp Clin Trials 2012;33:279-85.

44. Kusek JW, Ahrens A, Burrows PK, et al. Recruitment for a clinical trial of drug treatment for benign prostatic hyperplasia. Urology 2002:59:63-7.

45. Lee JY, Foster HE, McVary KT, et al. Recruitment of participants to a clinical trial of botanical therapy for benign prostatic hyperplasia. $J$ Altern Complement Med 2011;17:469-72.

46. Moinpour CM, Atkinson JO, Thomas SM, et al. Minority Recruitment in the Prostate Cancer Prevention Trial. Ann Epidemiol 2000;10:S85-S91.

47. Huynh L, Johns B, Liu S-H, et al. Cost-effectiveness of health research study participant recruitment strategies: A systematic review. Clin Trials 2014;11:576-83.

48. Silagy CA, Campion K, McNeil JJ, et al. Comparison of recruitment strategies for a large-scale clinical trial in the elderly. J Clin Epidemio 1991;44:1105-14.

49. Donovan JL, Peters TJ, Noble S, et al. ProtecT Study Group. Who can best recruit to randomized trials? Randomized trial comparing surgeons and nurses recruiting patients to a trial of treatments for localized prostate cancer (the ProtecT study). J Clin Epidemiol 2003;56:605-9.

50. Wallace K, Fleshner N, Jewett M, et al. Impact of a Multi-Disciplinary Patient Education Session on Accrual to a Difficult Clinical Trial: The Toronto Experience With the Surgical Prostatectomy Versus Interstitial Radiation Intervention Trial. Journal of Clinical Oncology 2006;24:4158-62.

51. Ford ME, Havstad SL, Davis SD. A randomized trial of recruitment methods for older African American men in the Prostate, Lung, Colorectal and Ovarian (PLCO) cancer screening trial. Clin Trials 2004:1:343-51.

52. Ngune I, Jiwa M, Dadich A, et al. Effective recruitment strategies in primary care research: a systematic review. Qual Prim Care 2012;20:115-23.

53. Menon U, Gentry-Maharaj A, Ryan A, et al. Recruitment to multicentre trials--lessons from UKCTOCS: descriptive study. BMJ 2008;337:a2079.

54. Hinshaw LB, Jackson SA, Chen MY. Direct mailing was a successful recruitment strategy for a lung-cancer screening trial. J Clin Epidemiol 2007;60:853-7.

55. Rooshenas L, Scott LJ, Blazeby JM, et al. The QuinteT Recruitment Intervention supported five randomized trials to recruit to target: a mixed-methods evaluation. J Clin Epidemiol 2019;106:108-20.

56. Juraschek SP, Plante TB, Charleston J, et al. Use of online recruitment strategies in a randomized trial of cancer survivors. Clin Trials 2017; 1740774517745829

57. Copeland RJ, Horspool K, Humphreys L, et al. Recruiting to a largescale physical activity randomised controlled trial - experiences with the gift of hindsight. Trials 2016;17:1-12.

58. UyBico SJ, Pavel S, Gross CP. Recruiting Vulnerable Populations into Research: A Systematic Review of Recruitment Interventions. J Gen Intern Med 2007;22:852-63. 
59. Gardner HR, Fraser C, MacLennan G, et al. A protocol for a systematic review of non-randomised evaluations of strategies to improve participant recruitment to randomised controlled trials. Syst Rev 2016;5:131.

60. Smith JA, Braunack-Mayer AJ, Wittert GA, et al. Qualities men value when communicating with general practitioners: implications for primary care settings. Med J Aust 2008:189:618-21.

61. The Clinical Trials Landscape in Australia 2006-2015: Australian New Zealand Clinical Trials Registry. http://www.anzctr.org.au/docs/Clin icalTrialsInAustralia2006-2015.pdf. (accessed 10 Jan 2018).

62. Elliott D, Husbands S, Hamdy FC, et al. Understanding and Improving Recruitment to Randomised Controlled Trials: Qualitative Research Approaches. Eur Urol 2017;72:789-98.
63. Pearson A, White H, Bath-Hextall F, et al. A mixed-methods approach to systematic reviews. Int J Evid Based Healthc 2015;13:121-31.

64. Treweek S, Bevan S, Bower P, et al. Trial Forge Guidance 2: How to decide if a further Study Within A Trial (SWAT) is needed [pre-print]. https://www.researchsquare.com/article/94854a88-d672-450db31a-b983828ccf49/v1 (accessed 11 Dec 2018)

65. Treweek S, Bevan S, Bower P, et al. Trial Forge Guidance 1: what is a Study Within A Trial (SWAT)? Trials 2018;19:139.

66. Healy P, Galvin S, Williamson PR, et al. Identifying trial recruitment uncertainties using a James Lind Alliance Priority Setting Partnership - the PRioRiTy (Prioritising Recruitment in Randomised Trials) study. Trials 2018;19:147. 